\title{
Radiopharmaceutical Dosimetry for Cancer Therapy: From Theory to Practice
}

\author{
Richard L. Wahl ${ }^{1}$ and John Sunderland ${ }^{2}$ \\ ${ }^{I}$ Mallinckrodt Institute of Radiology, St. Louis, Missouri; and ${ }^{2}$ University of Iowa, Iowa City, Iowa
}

$\mathbf{T}$ his supplement to The Journal of Nuclear Medicine includes 7 articles that address several of the critical facets of the current state of radiation dosimetry in radiopharmaceutical therapy. This supplement is designed to be a snapshot in time that attempts to address both the rapid progress and the challenges in applying patient-specific radiation dosimetry to guide radiopharmaceutical therapies. Six of the articles were generated by the Society of Nuclear Medicine and Molecular Imaging (SNMMI) Dosimetry Task Force led by Drs. Pat Zanzonico and George Sgouros, under the umbrella of the SNMMI's Research and Discovery Domain. As the perspective of the articles herein are largely based on practices in North America, an additional article is an invited perspective from Europe on the European approach to applying imaging-based dosimetry.

Looking back in time, ${ }^{131} \mathrm{I}$ has long been used for thyroid cancer therapies but normally has a sufficiently high therapeutic index that dosimetry is not required, though it is feasible. The first radiopharmaceutical therapy of cancer requiring dosimetry in the Food and Drug Administration (FDA) label was ${ }^{131} \mathrm{I}$ tositumomab, which was known commercially as Bexxar (1). The therapeutic regimen showed that it was feasible to generate a dosimetry-based dosing scheme for individual patients that could be widely disseminated. However, some viewed the dosimetry as too difficult ( 3 whole-body scans), and others felt the drug was too expensive. Long-term follow-up of randomized trials with the drug show significantly greater progression-free survival (PFS) than with standard therapies; nonetheless, the therapy was a commercial failure. Nearly 3 decades later, are we ready to revisit patient-specific dosimetry to drive radiopharmaceutical therapies?

Fueled by FDA approval of ${ }^{177}$ Lu-DOTATATE in 2018, and the promising published results of the ${ }^{177} \mathrm{Lu}-\mathrm{PSMA}-617$ phase 3 VISION trial earlier this year $(2,3)$, the nuclear medicine community is experiencing an unprecedented and palpable optimism for the future of the profession. In the wake of these developments, a slew of theranostic radiopharmaceuticals aimed at a variety of molecular targets and diseases are now rapidly entering early clinical trials, representing promise for downstream growth in the field. In parallel with these clinical developments, technologic and commercial advances in quantitative SPECT imaging, and development of sophisticated commercial internal radiation dosimetry software, are creating an environment whereby the vision of

Received Oct. 28, 2021.

For correspondence or reprints, contact Richard L. Wahl (rwahl@wustl.edu). COPYRIGHT @ 2021 by the Society of Nuclear Medicine and Molecular Imaging. DOI: 10.2967/jnumed.121.263273 accurate, reproducible personalized radiation dosimetry may be possible in the routine clinical practice of radiopharmaceutical therapy.

However, just because we can does not necessarily mean we should. This supplement begins to address whether radiopharmaceutical therapy is best performed as radioactive chemotherapy, for which the patient receives a standard dose that is determined from typical phase I-II dose escalation studies, and the toxicity profile from a given treatment is used to determine if subsequent administered activity levels should be adjusted upward or downward. This approach represents the "patient as the dosimeter" paradigm, with demonstration of physiologic toxicity as the readout of greatest relevance. However, because treatment decisions must be made promptly, this approach is difficult to use if toxicities are expected to be late in onset, potentially leading to underdosing of patients to avoid late toxicities. This supplement also addresses whether prospective imaging before a given treatment (or after, to verify dose delivery) can be used to guide patient-specific administered activity levels, which adjust for patient pharmacokinetics and which, in principle, would be expected to have greater efficacy and lower toxicity than a "one-dose-fits-all" approach.

It is clear that our radiotherapeutic armamentarium, current and future, contains a continuum of therapeutic indices. ${ }^{131}$ I therapy has historically had such a high therapeutic index that quantitative dosimetry would likely be a pointless exercise, resulting in little to no clinical benefit. ${ }^{177}$ Lu-DOTATATE and ${ }^{177}$ Lu-PSMA-617 both generate substantial survival benefit without patient-specific dosimetry-but how much better clinical performance might be achieved with dosimetry-guided optimization? And for new agents under current study, the therapeutic indices may be low enough that using patient-specific dosimetry is requisite to either qualify patients for the therapeutic radiopharmaceutical, or to titrate the therapeutic through image guidance. Clearly there are numerous questions still to both ask and answer.

Thus, we are currently facing the question "To D or not to D?" (where $\mathrm{D}=$ dosimetry) in no patients, selected patients, or all patients. We are probably not yet ready to answer this question, as it will require randomized trials to determine if dosimetry does, indeed, improve outcomes. On first principles, and based on decades-long experience with external-beam radiation therapy, it would seem that the ability to adjust dosing to a patient's specific pharmacokinetics and dosimetry would be more efficacious than giving repeated safe doses, which likely will result in underdosing the majority of patients. We cannot imagine giving external-beam therapy without dosimetry guidance. Will this eventually be the case for radiopharmaceutical therapies? And mission critical 
questions still remain, some scientific and some economic. What are the accuracies, variability, and reproducibility of our current dosimetry methodologies? (Short answer, we have substantial room for improvement.) What commercial tools are available? What is the outlook for reimbursement of dosimetry-related procedures? Under what circumstance does performing dosimetry have positive clinical impact?

The 7 articles in this supplement make a first-blush attempt to address these issues based on current information.

The first article, "Dosimetry for Radiopharmaceutical Therapy: Current Practices and Commercial Resources," summarizes the current quantitative paradigms for dosimetry calculations and provides detailed and up-to-date descriptions of currently available resources to perform dosimetry, including approved and evolving commercial software and standard radiation sources (4).

The second article, "Tumor Response to Radiopharmaceutical Therapies: The Knowns and the Unknowns," attempts to elucidate our current understanding (and lack thereof) of the subtle complexities underlying biologic responses of tissues to radiation. These include such topics as immune-mediated effects, radiobiologic mechanisms, tumor hypoxia, and dose rate effects (5).

Addressed in the third article, "Normal-Tissue Tolerance to Radiopharmaceutical Therapies, the Knowns and the Unknowns," is the critical topic of radiation-induced organ toxicities - the primary limiting factor in our dosing paradigm. This article reviews much of our current knowledge base, mostly derived from the high-dose-rate external-beam radiation therapy literature, which has potentially limited applicability to our low-dose-rate radiopharmaceutical therapy. The article clearly describes the significant lack of scientific organ radiation toxicity data for low-dose-rate treatments, for which cellular repair can play a significant role, and points out opportunities for careful future studies (6).

"An International Study of Factors Affecting Variability of Dosimetry Calculations, Part 1: Design and Early Results of the SNMMI Dosimetry Challenge," the fourth article, primarily describes the methodology associated with an international crowdsourced project attempting to quantify variabilities associated with discrete steps in the dosimetry workflow (7). Multisite variability projects have precedent in both the imaging and the therapy space $(8,9)$ but have not broken down individual steps as in the current challenge. In this progressive dosimetry calculation exercise, sites all over the world were provided identical multi-time-point DICOM SPECT/CT image data from 2 patients who underwent administration of ${ }^{177} \mathrm{Lu}$-DOTATATE and were asked to report stepwise dosimetric calculations. This article reports very early results (the challenge is still in progress) and demographics of respondents. Early data show there is considerable room for improvement in the consistency of dose estimation.

The fifth article, "Reimbursement Approaches for Radiopharmaceutical Dosimetry: Current Status and Future Opportunities," is perhaps the most pragmatic of the articles in this special edition and consists largely of a description of the various steps in the dosimetry workflow, and more importantly, current CPT codes that are likely appropriate to the various steps (10).

The current state of dosimetry (or lack thereof) in clinical practice today-largely in the United States but not exclusively-associated with approved radiopharmaceutical therapy agents is described in the sixth article. "Dosimetry in Clinical Radiopharmaceutical
Therapy of Cancer: Practicality Versus Perfection in Current Practice" discusses challenges associated with implementing dosimetry in the clinical space and limitations in available data currently supporting the use of dosimetry in standard clinical practice (11).

Finally, the seventh article, "Dosimetry for Radiopharmaceutical Therapy: The European Perspective," describes the European approach to dosimetry in clinical practice. In many ways the European nuclear medicine community has much more rapidly adopted the concept and practice of quantitative image-based dosimetry than the United States. This article describes the European dosimetry practice and details relevant position papers by the European Association of Nuclear Medicine (12).

Taken together, these articles provide a current state-of-theart understanding of the many elements of radiopharmaceutical therapy, highlighting the practical, the optimal, the knowns, and the unknowns, and provide valuable insights regarding commercial resources and billing approaches for dosimetry. The variability studies are of particular interest as they tell us that there are many opportunities to reduce variance and produce more uniform dosimetry.

We are hopeful these articles will provide a useful starting point and review for sites considering implementing dosimetry in their clinical practice or research operations. There is great interest and opportunity. The time to hesitate is through — there is much to do.

\section{REFERENCES}

1. Shadman M, Li H, Rimsza L, et al. Continued excellent outcomes in previously untreated patients with follicular lymphoma after treatment with CHOP plus rituximab or CHOP plus ${ }^{131}$ I-tositumomab: long-term follow-up of phase III randomized study SWOG-S0016. J Clin Oncol. 2018;36:697.

2. Strosberg J, El-Haddad G, Wolin E, et al.; for the NETTER-1 Trial investigators. Phase 3 trial of ${ }^{177}$ Lu-DOTATATE for midgut neuroendocrine tumors. $N$ Engl J Med. 2017;376:125-135.

3. Sartor O, de Bono J, Chi, KN, et al. Lutetium-177 -PSMA-617 for metastatic castration-resistant prostate cancer. N Engl J Med. 2021;385:1091-1103.

4. Capala J, Graves S, Scott A, et al. Dosimetry for radiopharmaceutical therapy: current practices and commercial resources. J Nucl Med. 2021;62(suppl 3): 3S-11S.

5. Sgouros G, Dewaraja YK, Escorcia F, et al. Tumor response to radiopharmaceutical therapies: the knowns and the unknowns. J Nucl Med. 2021;62(suppl 3): 12S-22S.

6. Wahl R, Sgouros G, Iravani A, et al. Normal-tissue tolerance to radiopharmaceutical therapies, the knowns and the unknowns. J Nucl Med. 2021;62(suppl 3): 23S-35S.

7. Uribe C, Peterson A, Van B, et al. An international study of factors affecting variability of dosimetry calculations, part 1: design and early results of the SNMMI dosimetry challenge. J Nucl Med. 2021;62(suppl 3):36S-47S.

8. O JH, Jacene H, Luber B, Wang H, et al. Quantitation of cancer treatment response by ${ }^{18}$ F-FDG PET/CT: multicenter assessment of measurement variability. $\mathrm{J} \mathrm{Nucl}$ Med. 2017;58:1429-1434.

9. Mora-Ramirez E, Santoro L, Cassol E, et al. Comparison of commercial dosimetric software platforms in patients treated with ${ }^{177}$ Lu-DOTATATE for peptide receptor radionuclide therapy. Med Phys. 2020;47:4602-4615.

10. Graves SA, Bageac A, Crowley JR, Merlino DAM. Reimbursement approaches for radiopharmaceutical dosimetry: current status and future opportunities. $\mathrm{J} \mathrm{Nucl}$ Med. 2021;62(suppl 3):48S-59S.

11. Pandit-Taskar N, Iravani A, Lee D, et al. Dosimetry in clinical radiopharmaceutical therapy of cancer: practicality versus perfection in current practice. $J \mathrm{Nucl} \mathrm{Med}$. 2021;62(suppl 3):60S-72S.

12. Lassmann M, Eberlein U, Gear J, Konijnenberg M, Kunikowska J. Dosimetry for radiopharmaceutical therapy: The European perspective. J Nucl Med. 2021; 62(suppl 3):73S-79S. 